\title{
vert. 13952
}

\section{AUTORADIOGRAPHIC ENHANCEMENT OF POLAROID FILMS}

November 1978

Final Report for Period 18 April to 17 Oclober 1978

By: R. G. Monning and R. R. Pettijoln

Preperedtor:

Lawrence Livermore Laboratory

P.O. Box 808

Livermore, California 94550

Attn: Dr.R.C.Woingart

Order No. 1263309

SPI ProjectPYD-7420

SFil International

333 Raventwwrood Avenuse

Menlo Park, California 94025

(415) 326-6200

Crble: SAIINTL MNP

TWX: $810-373-1246$ 


\section{AUTORADIOCRAPHIC ENHANCEMENT OF POLAROID FILMS}

November 1978

Final Report for Porlod te April to 17 Octobar 1978

By: R. C.Munning and A.R. Pottliohn

Preperadtor:

Lawrence Liwermoro Leboratory

P.O. Box 808

Livermore, Cellifomia 94560

Atth: Dr.R. C. Wolngert

Order No. 1263600

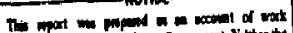

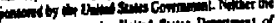

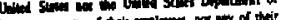

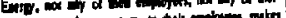

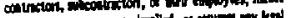

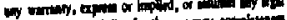

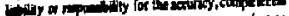

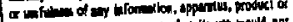

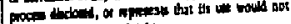

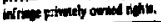

SRIProject Pro-7420

Aporoved:

Q. A. Abrthemeon, Director Poulter Lebovitory

MISTER

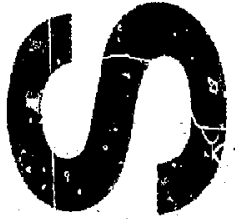

P.J.Jorgunen, Vice Preaident Physicel end Lib 8ctences: 


\section{CoNHEATs}

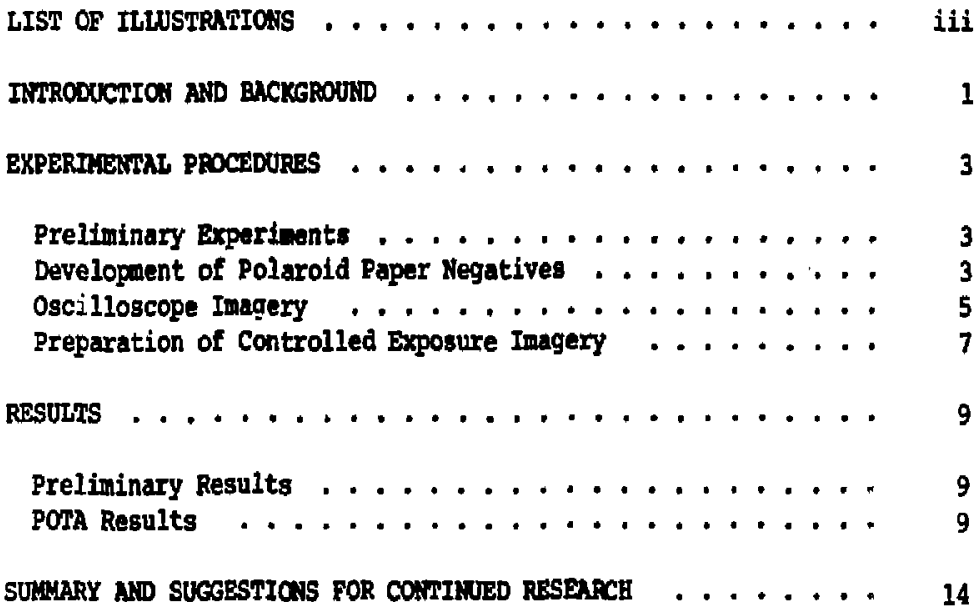


1. Schematic of Circuit Uned to Generate Standard

Oscilloscope Trace ................ 6

2. Standard 0scilloscope Trace used in Quantitative Image

Bnhancement Exporiments ............ 8

3. Comparison of Polaroid Exposure Sequence and Autoradiographic Enhancement of Polarold Type $47 \mathrm{Film} \ldots \ldots$

4. Comparison of Polaroid Exposure Sequence and Autoradiographic Enhancenent of Polaroid Type 57 Film ......... 11

5. Camparison of Polaroid Exposure Sequence and Autoradiographic Enhancement of Polaroid Type 410 Fill ........ 13

TABLE

1. Effective Polaroid Film Speed Increase Using Autoradiographic Hethods .................. 


\section{INTRODOCTION AND BATTERDOND}

Under a six-month contract to Lawrence Livermore Laboratory (LLC), SRI International has investigated the application of radiotracer photographic image enhuncement (PIE) techniques to increase the photographic speed of three Polarold Files. SRI has a state-of-the art capability in the field of nondestructive photographic image enhancement of original negatives. With these procedures, we have successfully increased optical density, contrast, and resolution of photographic imagery, and thereby increased the information derivable from original negatives. The present LLI con ract represents our first attempt at PIE treatment of Polaroid fillns.

Polaroid files pose unique experimental difficulties, principally because of their paper negatives, which contain many chemicals not found in conventional photographic negatives. These chenicals are necessary for the unique polaroid development process. Radiotracer PIE techniques are currently the only method of enhancing Polaroid inagery because other methods, such as digital processing or increased contrast printing, depend on light transmission through the negative. Since the Polaroid negative is opaque, these other methods are not applicable.

current difficulities with polaroid filpa include the recording of transient oscilloscope traces, which are too fast for the film. In many cases these traces can be the output signal from-an expensive, hard-to-reproduce experinent. If the trace image is not severly underexposed, radiotracer PIE nethods may recover the invisible but existing inage. This research is directed toward increasing the effective Polaroid film speeds to allow recovery of such imagery. 
The coupleted research included the following tasks:

- Preparation of controlled calibration inagery on Polaroid filie types 17 (ASA 3000), 57 (ASi 3000), and 410 (ASA 10,000), developed uaing the Polaroid developer, Bastman rodak (EX) D-19, or POIS.

- PIE procussing of the controlled calibration imagery, using a variety of toning techniques and isotopes to determine the bent procedure.

- Evaluation of the enhancement capabilities and effective speed increase achievable using PIE techniques.

This report contains a general discussion of the experimental methods used and results obtained on SRI-prepared cmtrolled imagery. The final section includes a sumary and suggestions for continued rasearch. 


\section{EXPERILETIAL PROCEDURES}

\section{Preliminary Experiments}

Preliminary experiments focused on the feasibility of simply applying PIE techniques to Polaroid paper negatives that had been developed according to standard polaroid procedures. This approach resulted in autoradiographs with unacceptably high fog levels. These high fog levels were caused by the chenicals used in the Polaroid developing method. The fog levels were lowered by immediately fixing the paper negative in $\mathrm{KR}$ Rapid Fixer after developing the film, as described below. However, altemative developers gave even lower fog levels and consequently better image enhancement. Procedures were developed to bypass the normal polaroid development procedure, develop the Polaroid negative using darkroom chemical processing, ard autoradiographically enhance the resulting inage.

\section{Development of Polaroid Paper Negatives}

Besides the Polaroid developer, two other developers were investigated: EK D-19 and POTA. EK D-19 was chosen because it is a frequently used high-contrast, clean-working developer. It is recommended by Kodak for continuous tone work that requires higher-than-normal contrast. POTA was originally formulated to provide a developer than yielded low fog and an extended toe region in a plot of log exposure versus optical density. An extended toe region in the Polaroid paper negative should allow greater autoradiographic enhancement since intensification of this region of image ressults in an effective speed increase. 
Three film sets were prepared. Film set 1 consisted of films that were exposed and developed ancoreling to Polaroid instructions. The film packets were then opened in a dackened rocm, and the paper negatives were irmersed in EK Rapid Fix for 5 minutes and washed in running deicnized water for 30 minutes.

Film set 2 consisted of film developed in EK D-19. The holder for Polaroid type $57 \mathrm{film}$ was modifled by rewoving the steel and rubber rollers. This removal prevented the breaking of the chemical pod in the film packet and thereby prevented the film from being conventionally Polaroid developed.' After the exposures were made, the packets were opened in total darkness/ The pod was detached from the paper negative, and the negative was processed in EK D-19 for 3 minutes, in 18 acetic acid stop bath for 30 seconds, in ER Rapid Fix for 3 minutes, and then washed in running water for 30 minutes. For Polaroid types 47 and 410 , $4 \times 5$ Graflex film holders were modified by inserting a piece of clear plastic to hold the Polaroid negatives flat. This was recessary because the paper negatives had a tendency to curl. The paper negative was then cut into sections of the correct size and inserted behind plastic. After exposure, the negatives were processed in D-19 for 3 minutes, acetic acid stop bath for $30 \mathrm{sec}$, EK Rapid Fix for 3 minutes, and then washed in running water for 30 minutes.

For film set 3, the various types of Polaroid film were handled in the same manner as set 2, except that POTA was used as the developer. POTA consists of $37 \mathrm{Na}_{2} \mathrm{SO}_{3}+0.157$ phenidone. Development time was 15 minutes. Fresh POTA was prepared daily because it oxidizes quickly and cannot be stored without special precautions. 


\section{Oscilloscope Imagexy}

Precise evaluation of image enhancement and film speed increases requires controlled imagery. We wanted a test image that would simulate an oscilloscope trace that might be received during an actual experiment and that would also allow quantitative speed increase evaluation.

To satisfy these conditions, we developed a system for placing multiple traces of variable optical density on an oscilloscope. Since the optical density of any trace on an oscilloscope is a function of the scan speed, the system was designed to sequentially induce different waveforms with the same period and at diverse amplitudes in a retrace mode on the oscilloscope. A block diagram of this circuit is shown in Eigure 1. The square wave generator produces a wave train with a period of 1 ins ( $1 \mathrm{kHz}$ frequency). The frequency (f) is determined by

$$
f=\frac{1.44}{\left(R_{1}+2 R_{2}\right) C_{1}}
$$

where $R_{1}$ and $R_{2}$ are resistances and $C_{1}$ is the capacitance. In our application $R_{1}=3.0 \mathrm{~K}, R_{2}=5.7 \mathrm{~K}$ and $C_{1}=0.01 \mu \mathrm{F}$. This wave train is transferred to an integrator where voltage out $\left(e_{0}\right)$ is determined by:

$$
e_{0}=\frac{1}{R_{3} c_{2}} \int_{0}^{t} v_{\text {in }} d t
$$

where $R_{3}=10 \mathrm{~K}, c_{2}=1 \mathrm{mfd}, v_{\text {in }}$ is the input voltage and the time. Thus the output waveform from a square wave input is a triangular waveform.

The output of the square wave generator is also sent to the sequentially operated analog switch. When analog switch 1 is active, the square wave appears on the output and the proportional gain implifier is disabled. When analog switch 2 is active, the triangular waveform appears at the output and the proportional gain amplifier is set for unity gain. When analog switches 3,4 , and 5 are activated, the proportional gain anplifier is set at $\times 2, \times 10$, and $\times 1000$ gain, respectively, in sequence. Thus, the writing speed is varied from 1 volt/ws 


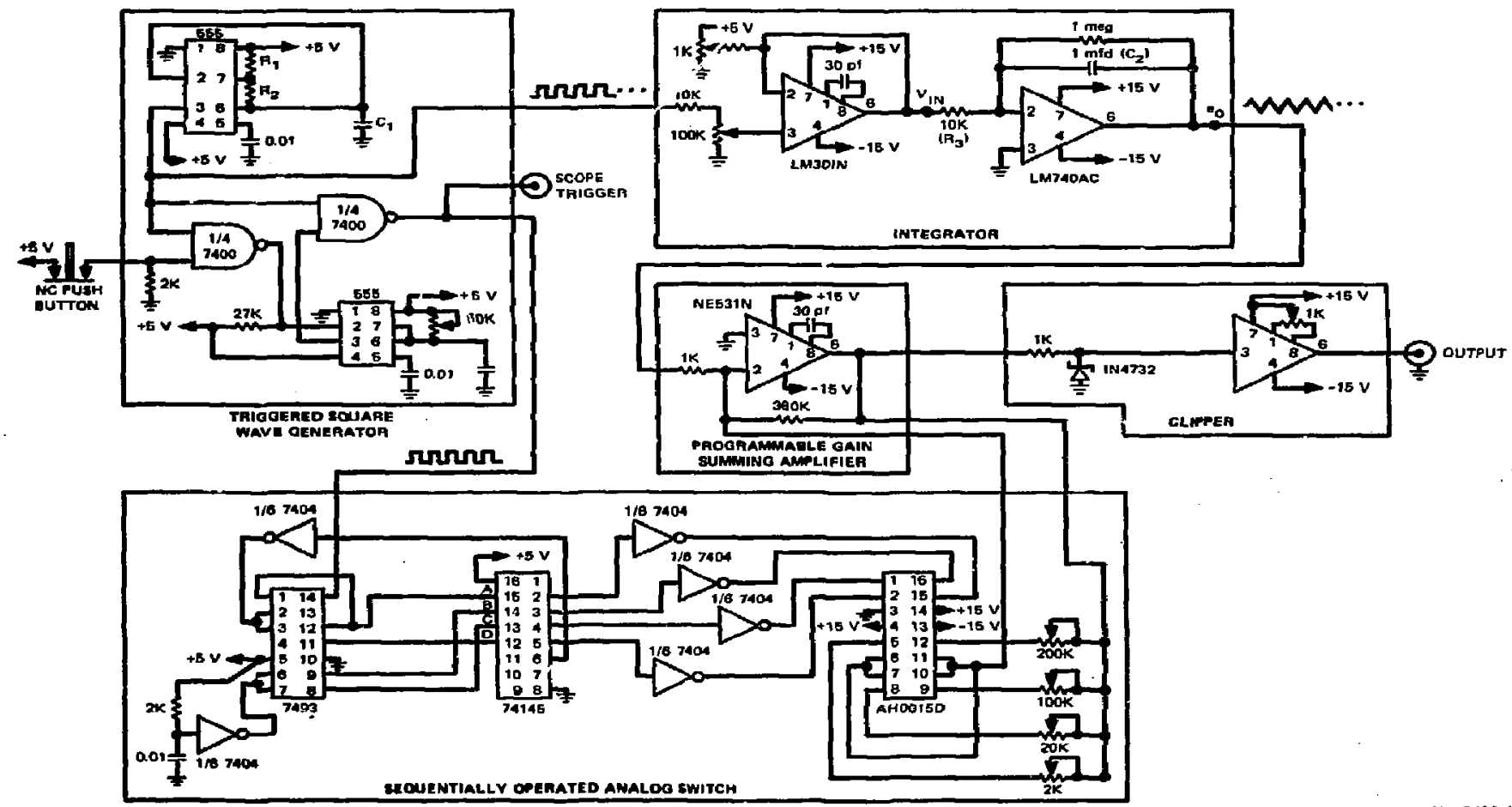

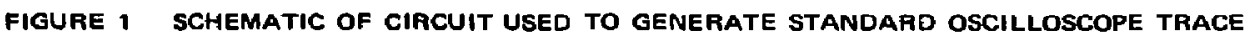


to he maximum slew rate of the proportional gain amplifier, or alproximacejy 50 volts/hs. The function of the clipper is to contain all the waveforms within a fixed maximum amplitude.

The scale is triggered earh time the positive slope of the square ware aptears. Thus, with the prcper setting of the time base on the 1.:Llloscope, a!l the waveforms can be superimposed. Wo used a Tekryonix Iype 555 dual-beam oscilloscope with Tektronix typo $53 / 54 k$, Jus ins, Tektronix tyle $21 \mathrm{~A}$ or type 22A time base units, and a Tektronix Modkl C-27 oscilloscope camera equipted with a Wollensak $75 \mathrm{~mm}$ f/l." oscilloantor $?: 0.7 x$ lens. A typical oscilloscope trace ls showl as Fiqure $\therefore$ in single exposure such as that in Figure 2 contains magry with an: intcisisity dytamic range of several thousand-fold.

\section{Egaration of Controlled Exposure Imager:}

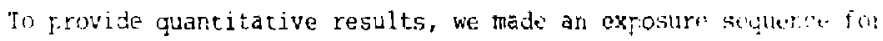
each film type. The exposure was varied throughout the sequence by char: 1ng the aperture and/or the exposure riuration. Exyosures weye made over a range of about a thousand. The resulting exposure stupenctic tive allowed a direct visua, determination of the effective film speed morease. Ths visual comparison method, using reflected ljght, is analogous to using a step wedge to provide sensitometric evaluation of a : ansparent filn's speed, using transmitted light. 


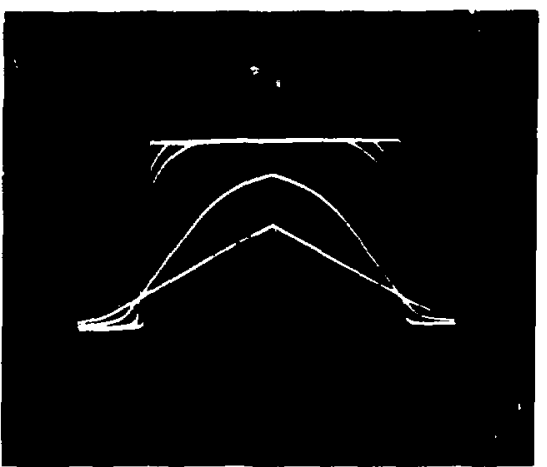

4. $19.7420-2$

FIGURE 2 STANDARD OSCILLOSCOPE TRACE UST D M SUMMTI:ATIVE MAGE ENHANCEMENT EXPERIMENTS 
RESULTS

\section{Preliminary Results}

During the initial qualitative phase of this investigation, all three film types were evaluated for each of the three development. procedures, Radiotoning procedures involving the radioisotopes nickel63, iodine-125, and sulfur-35 were used. Although type 410 film exhibi ted significant enhancement regardless of the developer formulation, these early experiments showed that the best image enhancement was obtained with the POTA developer, regardless of film type. Accordingly, we decided to concentrate on the three film types developed in PoTA.

\section{POTA Results}

Figure 3 compares the Polaroid exposure sequence for type $47 \mathrm{film}$ and an autoradiograph resulting from the sulfur-35 toning of an original paper negative having a relative exposure value of 1 . The autoradiograph corresponds to an original negative having a relative exposure value of more than 2 but less than 4 . We have thus increased the effective photographic speed of polaroid type $47 \mathrm{film}$ by a factor of 2 to 4 . Autoradiographs were prepared using EK type SR or type AA $x$-ray film as the receiving film.

Figure 4 shows similar results for type $57 \mathrm{film}$. Once again we toned an original negative having a relative exposure of 1 . The resulting autoraciograph is shown in the upper right in Figure 4. The photograph ic sfeed has again been increased 2 to 4 times that of the original. 

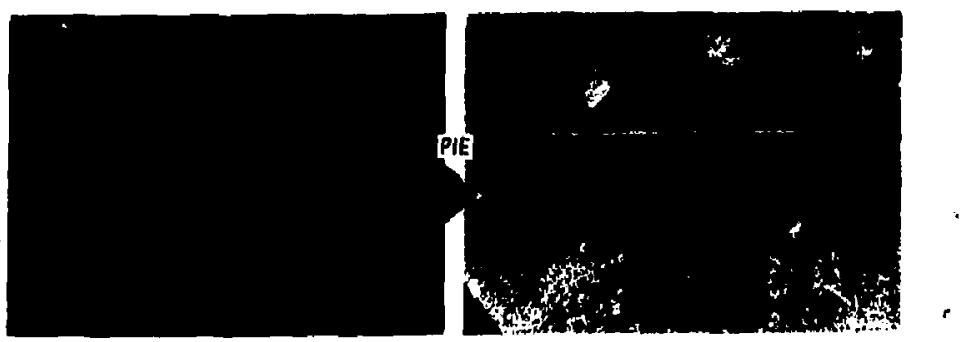

2

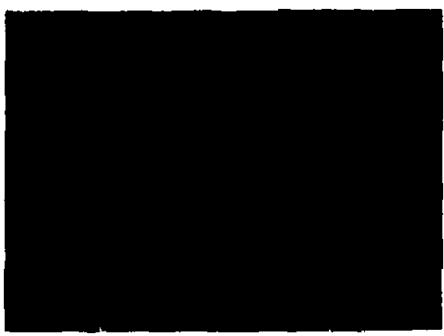

4
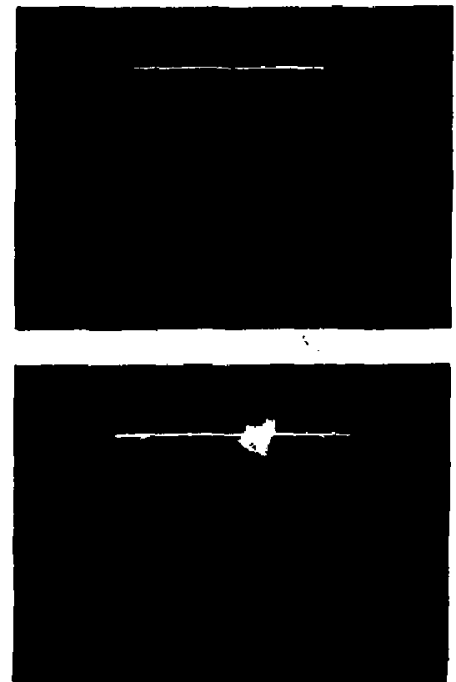

8

$M P-74 \times 0-3$

FIGURE 3 COMPARISON OF POLAROID EXPOSURE SEQUENCE AND PIE AUTORADIOGRAPHIC ENHANCEMENT OF POLAROID TYPE 47 FILM 

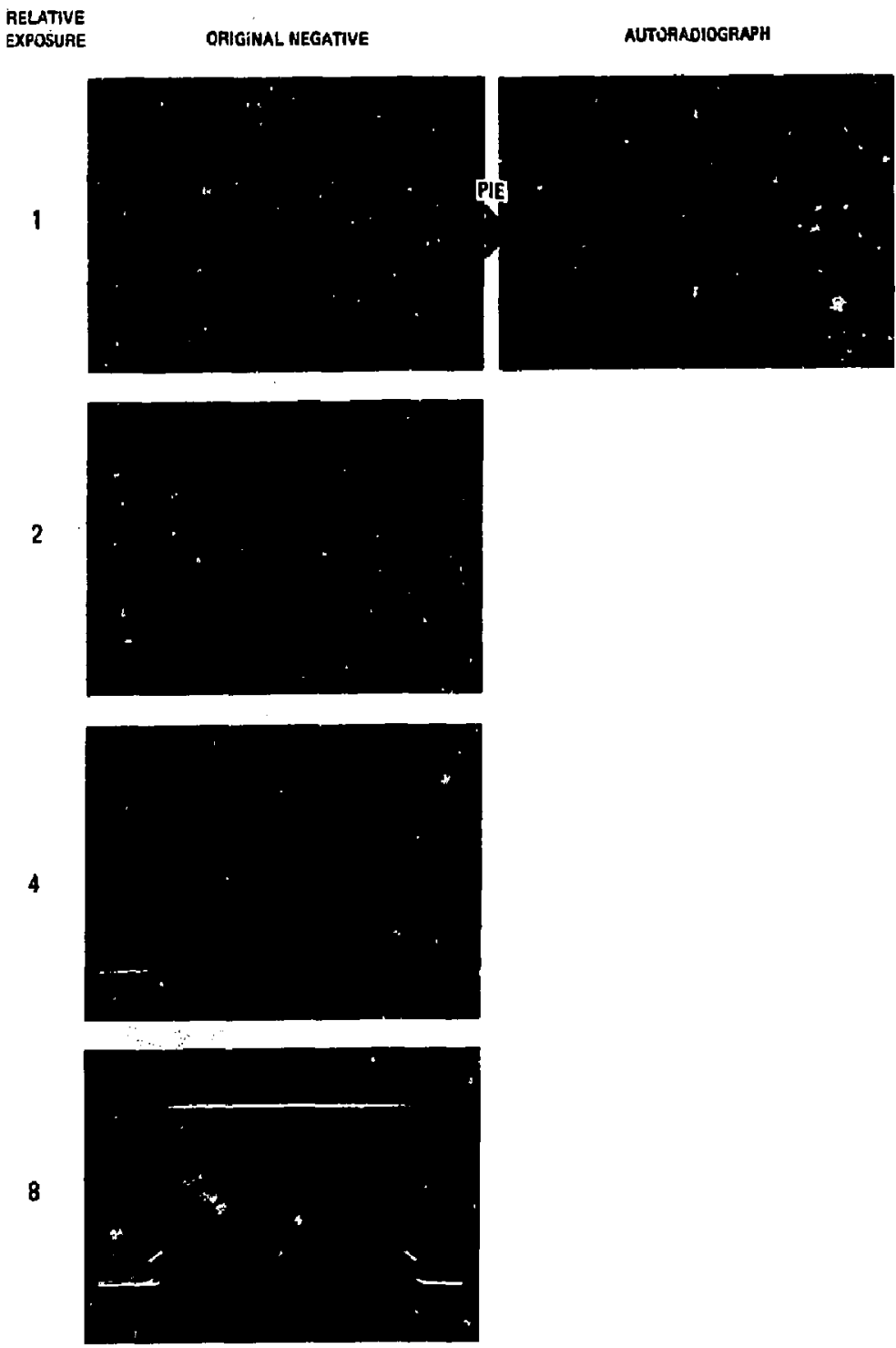

M. $7420-4$

FIGURE 4 COMPARISON OF POLAROID EXPOSURE SEQUENCE AND PIE AUTORADIOGRAPHIC ENHANCEMENT OF POLAROID TYPE 57 FILM 
Figure 5 contains filn type 410 results. A speed increase of more than 2 is again realized. Although we also toned duplicate original negatives with Ni-63 and I-125, S-35 yielded similar results in this preliminary study. Standard toning wethods were used, and no attermt was made to optimize them for these particular film types.

The 5-35 procedure is as follows: The negatives were first washed in deionized water for 10-20, minutes. They were then radiotoned by agitation in a S-35 labeled thioured aqueous solution for 2 hours. The $\mathrm{pH}$ of the solution was 11.5 and was adjusted with $18 \mathrm{~K}_{2} \mathrm{CO}_{3}$. The thiourea was present at a concentration of $0.13 \mathrm{mCi}$. S-35 labeled thiourea was purchased from New England Nuclear at a specific activity of $4 \times 10^{4}$ cilg. Following the radiotoning, the negative was washed in deionized water for 10 minutes, in 30 methanol for 10 minutes, and in deionized water for 10 minutes. The negative was then air dried.

Autoradiographs were prepared by placing the $x$-ray-sensitive receiving film in contact with the toned paper negative in a vacuum cassette. Autoradiogrpahic exposures varied from a few hours to a few days depending on the level of radioactivity in the toned negative. 


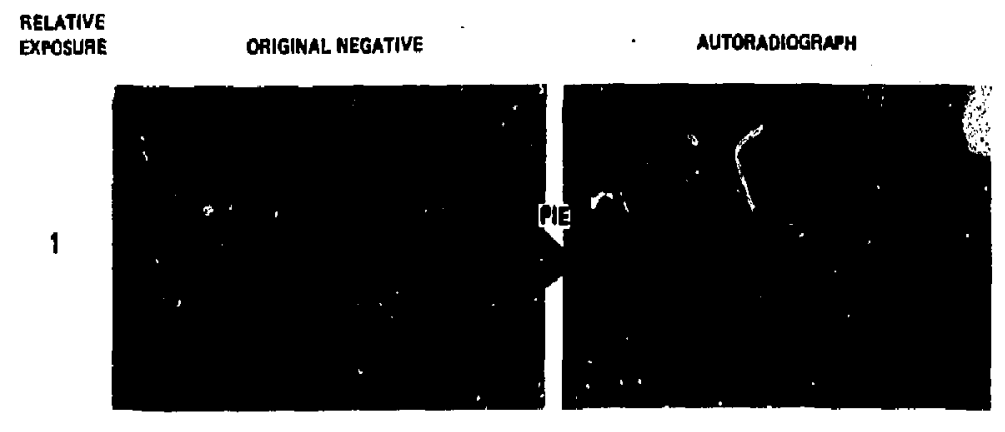

2

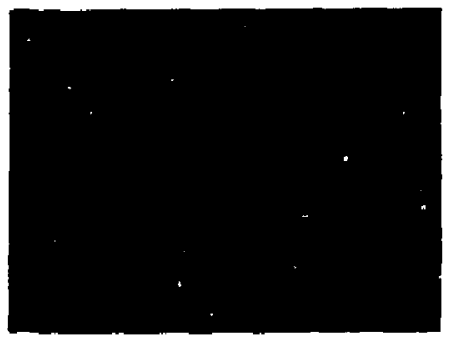

4

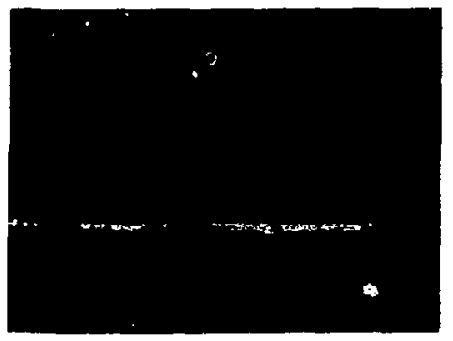

8

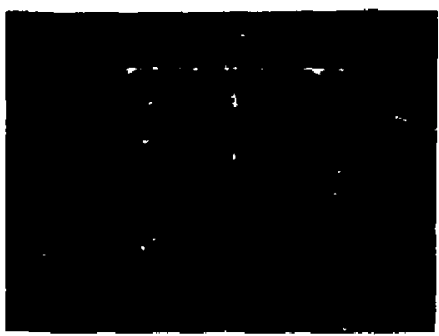

MP-7420-6

FIGURE 5 COMPARISON OF POLAROID EXPOSURE SEQUENCE ANU PIE AUTORADIOGRAPHIC ENHANCEMENT OF POLAROID TYPE 410 FILM 
SLRMARY AND SUGGESTIOUS FOR CONTINUED RESEARCH

SRI International has completed a preliminary study to experimentally evaluate the effective speed increases achievable with Polaroid film types by the application of antoradiographic methods. The procedures developed for Polarold film types 47, 57, and 410 increased their effective speeds 2 to 4 times that obtaired using conventional Polaroid procedures, as sumarized below.

Table 1

\begin{tabular}{ccc}
$\begin{array}{c}\text { Polaroid } \\
\text { Film Type }\end{array}$ & $\begin{array}{c}\text { Polaroid Film } \\
\text { Speed (ASA) }\end{array}$ & $\begin{array}{c}\text { Effective Polaroid } \\
\text { Film Speed Using }\end{array}$ \\
\cline { 2 - 3 } 47 & 3,000 & 6000 to 12,000 \\
57 & 3,000 & 6000 to 12,000 \\
410 & 10,000 & 20,000 to 40,000
\end{tabular}

Type $410 \mathrm{film}$ is currently the fastest comercially available photographic recording medium. However, applying auotradiographic technology to type 410 photographic applications extends the film's capability so that it can record events that occur 2 to 4 times faster or in which the light intensity is 2 to 4 times less than was previously possible.

Additional research and devleopaent in the following areas should be undertalen to obtain further increases in effective film speed of Polaroid films: 
(1) Negative pretoning treatments need to be formulated to allow direct treatment of conventionally developed Polaroid negatives.

(2) Radiotoning methods should be optimized for Polaroid films.

(3) An optimum procedure should be developed to aid in implementing this new capability. 